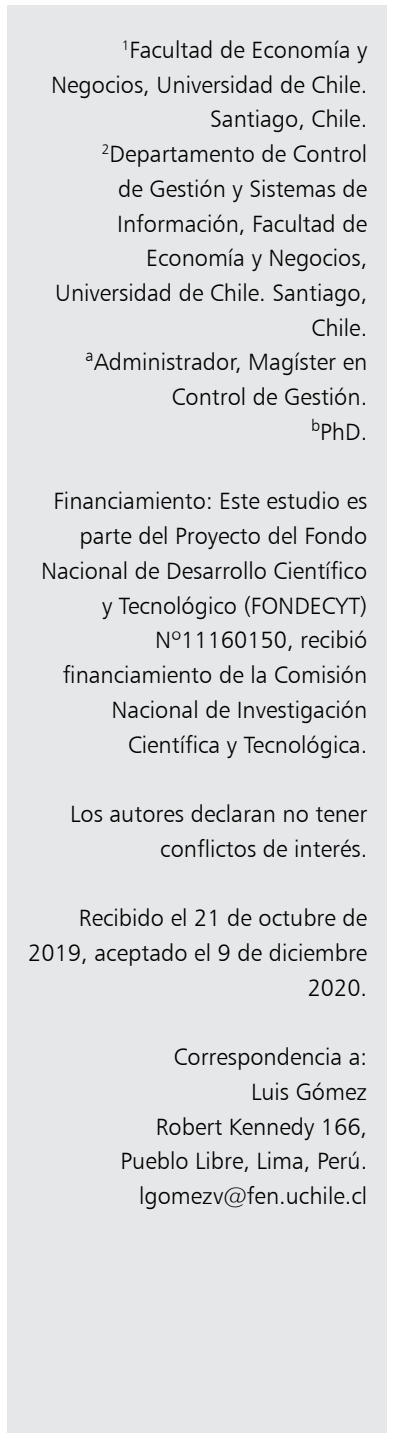

\section{Vigilancia del acceso a la salud en Chile: un sistema de indicadores para monitoreo multidimensional}

\author{
LUIS GÓMEZ ${ }^{1, a}$, ALICIA NÚÑEZ 2,b
}

\section{A system aimed at monitoring healthcare access in Chile}

Background: A health system is successful when it maintains a healthy population and provides health services that are effective, equitable and safe. Aim: To design a system based on specific indicators to monitor equity in health care access in Chile. Material and Methods: Primary information about five dimensions of access to health was collected, namely health policies, characteristics of the health system, characteristics of the population at risk, utilization of health services and consumer satisfaction. Subsequently, inequalities in access between different social groups were identified. Finally, after collecting and filtering access indicators used in other systems and existing literature, the most relevant ones were selected to monitor each identified barrier. Results: A system composed of 26 indicators, classified in the five dimensions of the framework was devised. It allows us to monitor those barriers with a greater impact on the population. For some specific indicators, population groups were disaggregated to carry out specific surveillances. Conclusions: The design of a multidimensional monitoring system for health access allows us to complement measures usually focused on a specific concept of access (such as utilization, coverage, etc.) with other dimensions. It includes those barriers that are relevant for the Chilean population. It also allows comparisons with other health systems and the generation of evidence to improve public policies.

(Rev Med Chile 2021; 149: 62-75)

Key words: Health Services Accessibility; Health Equity; Healthcare Disparities.
U n sistema de salud es exitoso cuando logra mantener a su población sana, provee servicios de salud efectivos, seguros, equitativos y eficientes, y es justo, brindando atención sin discriminación a todas las personas ${ }^{1}$. Chile, se ha enfocado en las últimas décadas, en mejorar los indicadores básicos de salud, realizando actividades conducentes a reducir la desigualdad en el acceso a la salud ${ }^{2}$. Entre las acciones más destacadas se encuentran la creación de la Superintendencia de Isapres (ahora extinta), el establecimiento del Régimen de Garantías Explícitas en Salud, además de un aumento de infraestructura, equipamiento médico y salarios del sector salud ${ }^{2,3}$. No obstante, pese a los esfuerzos realizados, la inequidad es un problema vigente, observable, por ejemplo, en las diferencias en la disponibilidad de personal médico entre los subsectores público-privado, teniendo este último, una disponibilidad superior en por lo menos cinco veces ${ }^{4,5}$. Elementos como este, que impiden que una persona obtenga un acceso a la salud satisfactorio, son denominados barreras de acceso a la salud, y constituyen un problema en el alcance de una cobertura universal ${ }^{6}$.

Entre las acciones para enfrentar dicho problema, se encuentra la evaluación y medición del nivel 
de acceso a la salud presente en la población. Los sistemas de medición de desempeño son usados para la mejora de los programas de salud mediante el monitoreo de la implementación y los resultados de estos, así también para la asignación de responsabilidades ${ }^{7}$. Dentro de dichos sistemas, los indicadores juegan un papel primordial dado que monitorean los resultados de salud, los servicios integrales, las inequidades y los determinantes sociales de la salud, datos que, preferentemente, deben estar desagregados para facilitar el monitoreo de los avances en torno a la equidad ${ }^{8}$. Así, indicadores de acceso a la salud cubren aspectos como la cobertura de aseguramiento en la población, la disponibilidad y accesibilidad física o el nivel de utilización ${ }^{9}$, los cuales pueden variar entre realidades y sistemas de salud, dependiendo de sus prioridades ${ }^{9}$. Estos resultados permiten evaluar si la sociedad cumple con sus responsabilidades en organización y prestación de servicios de salud, así como movilizar presiones sociopolíticas a fin de garantizar condiciones que eleven los niveles generales de salud de la población ${ }^{10}$.

De este modo, el presente artículo propone un sistema de indicadores que permita monitorear los niveles de acceso a la salud en Chile, así como identificar potenciales inequidades en salud entre los distintos grupos de la sociedad, con base en las opiniones brindadas por comunidades pertenecientes a tres regiones del país: Antofagasta, Biobío y Metropolitana de Santiago. El objetivo de dicho sistema consiste, bajo un enfoque de control de gestión, tanto en proponer un instrumento de monitoreo que pueda ser utilizado para vigilar el alcance de objetivos de acceso a la salud, así como en proveer de información para análisis, diseño o mejora de políticas públicas actuales o nuevas.

\section{Materiales y Métodos}

Se considera, un concepto multidimensional de acceso a la salud según el modelo de cinco dimensiones propuesto por Aday y Andersen ${ }^{11}$ :

- Política de Salud: Evalúa los esfuerzos realizados en torno al diseño de políticas públicas destinadas a una mejora del acceso a la salud por parte de la población, políticas que, a su vez, tienen un impacto en el resultado del resto de dimensiones.

- Características del Servicio de Salud: Incluye todos los elementos involucrados en la entrega de cuidados médicos a los usuarios, clasificados en dos grupos principales: recursos y organización.

- Características de la Población: Considera aquellos elementos que son determinantes de una potencial utilización de los servicios de salud, siendo clasificados en tres grupos: De predisposición, que existen antes de la ocurrencia de un episodio de enfermedad y que describen la propensión a utilizar los servicios de salud (por ejemplo: edad, sexo, pertenencia a pueblo originario, ocupación, educación, actitudes y comportamientos relativos a la salud); los facilitadores, que incluyen los medios que permiten que los individuos hagan uso de los servicios de salud (por ejemplo: ingreso, pertenencia a un sistema de aseguramiento de salud, residencia en zona rural o urbana); y de necesidad, referido al nivel de enfermedad, representando la causa inmediata de uso de atención médica (por ejemplo: existencia de enfermedades crónicas u obesidad diagnosticadas).

- Utilización de Servicios de Salud: el acceso real de la población al sistema de salud, donde convergen las necesidades de los pacientes y el sistema de salud en $\mathbf{s i ́}^{12}$. Puede caracterizarse en términos de tipo de servicio, lugar en que se realizó el servicio, propósito y el intervalo de tiempo involucrado.

- Satisfacción del Usuario: analiza el acceso real a través de medidas subjetivas expresadas por parte de la población acerca de la calidad del servicio recibido.

De esta forma, se analiza el acceso siguiendo un modelo lógico de entradas (Política de Salud), procesos (Características del Servicio de Salud y de la Población) y resultados (Utilización de los servicios de salud por parte del usuario y la Satisfacción de este). Con base en dicho modelo, se llevó a cabo un estudio descriptivo longitudinal cuya primera etapa, consistió en recopilar, a través de encuestas representativas, información que permita identificar el nivel de acceso a la salud de la población, así como definir qué elementos ésta considera como las principales barreras de acceso a la salud. El estudio partió por seleccionar tres regiones (Antofagasta, Metropolitana de Santiago y Biobío), considerando su tamaño poblacional y la cantidad de grupos étnicos presentes. Para cada región, tomando como marco muestral el Censo 
de Población y Viviendas del 2002, se realizó un muestreo estratificado de individuos -siendo este la unidad de análisis- representativo a nivel regional y en los estratos urbano-rural que la componen, muestra que fue contactada seleccionando aleatoriamente tanto los hogares como los individuos pertenecientes a estos. En total, se recopilaron 1.885 encuestas en las tres regiones consultadas, abarcando 42 localidades rurales y 231 localidades urbanas. El cuestionario consultado a la muestra incluyó preguntas abiertas y cerradas, además de preguntas de tipo demográfico y datos personales, preguntas relacionadas al acceso a la salud según cada una de las cinco dimensiones del modelo de acceso ${ }^{11}$. Para la elaboración de las preguntas se utilizó un modelo ontológico que tuvo como objetivo mapear las distintas barreras o facilitadores de acceso a la salud que el modelo estudiado propone, con base en la combinación de tipo de recursos involucrados, tipo de acceso, tipo de atención médica, tipo de personal prestador de salud involucrado y tipo de población usuaria ${ }^{13}$. El resultado final fue un listado de preguntas divididas en siete módulos.

Tras la capacitación a los entrevistadores y la realización de las encuestas, la información recabada fue analizada a fin de determinar qué elementos de cada dimensión representaban barreras relevantes de acceso a la salud para la población. Para asignar el nivel de importancia que la muestra consultada otorga a cada barrera de acceso a la salud, se hace uso de las conclusiones de un estudio paralelo, realizado con la misma información primaria, que utiliza la teoría de utilidad multiatributo para obtener una función de utilidad numérica cuyo valor (entre 0 y 1 ) determina una mayor o menor desutilidad causada por dicha barrera a la población. Se consideraron como barreras relevantes aquellas cuyos valores de desutilidad fueron más cercanos a 1 .

Adicionalmente, este estudio busca que el acceso a la salud sea visto bajo un enfoque de equidad en el que las diferencias en salud entre individuos, de existir, no debieran estar determinadas por presencia o ausencia de factores como raza, nivel de ingresos, cobertura del seguro de salud, u otros factores geográficos o sociodemográficos, sino que los servicios de salud deban ser distribuidos con base en las necesidades de salud de los individuos ${ }^{14}$. De esta forma, en paralelo, se realizó un exhaustivo análisis literario de factores (tales como edad, género, residencia en zonas rurales o pertenencia a un grupo étnico, por mencionar algunos) que podrían ser causantes de inequidad para cada una de las barreras al acceso identificadas como relevantes por la población. Se identificaron aquellos susceptibles de aparición dada las características de la muestra y se comprobó, mediante análisis estadísticos (pruebas de diferencia de proporciones, regresión logística, t-Student) realizados con la información obtenida de las encuestas, la existencia o no de inequidad en el acceso para cada barrera entre distintos grupos de la población. De existir tal inequidad, se sugirió desagregar el indicador de monitoreo (por ejemplo, de existir diferencia estadísticamente significativa en los tiempos de traslado al servicio de emergencia entre población urbana y rural, el indicador resultante será desagregado en dos grupos). Asimismo, se realizó la revisión de más de 100 indicadores de monitoreo de acceso a la salud-tanto aquellos utilizados en otros sistemas de salud como los provenientes de la literatura en publicaciones especializadas-los cuales fueron clasificados según la dimensión del modelo de Aday y Andersen ${ }^{11}$ a la que pertenecían. De esta manera, se obtuvo una selección final de 26 indicadores, considerados los más idóneos para monitorear cada barrera. Este grupo final de indicadores incluye indicadores propuestos de forma original para este estudio, en caso de que no se encontrara un indicador para alguna barrera particular en otro sistema de salud o en la literatura. A cada indicador formulado se le asignó una meta de referencia a fin de contar con un estándar de desempeño que permita direccionar los esfuerzos del sistema de salud hacia una mejora en los niveles de acceso para la población chilena, además de ser un elemento motivante hacia la acción ${ }^{15}$. Las cifras para las metas de cada indicador fueron asignadas considerando, en primer lugar, una cifra objetivo de referencia que una entidad supranacional (OMS u OCDE) haya fijado para el mismo indicador. De no contar con dicha cifra disponible, se tomó en cuenta una cifra objetivo fijada por otro sistema de salud de un país comparable a Chile. De tratarse de un indicador medido únicamente de forma local en Chile, la meta objetivo fue establecida considerando tendencias de estadísticas previas. Finalmente, de tratarse de un indicador propuesto originalmente para esta investigación, se determinó una cifra razonable, al no contar con información base de desempeño. 
Vigilancia del acceso a la salud en Chile - L. Gómez et al

La Figura 1 resume la metodología llevada a cabo en este estudio.

\section{Resultados}

Según la metodología considerada, el primer paso fue la identificación de las barreras de acceso con mayor impacto en la población, resumidas en la Tabla 1.
El siguiente paso consistió en la asignación de indicadores para cada barrera. A cada indicador se le asignó una cifra meta de referencia, siguiendo la metodología planteada. La Tabla 2 resume los indicadores considerados para monitorear los niveles de acceso a la población para cada barrera identificada, así como las metas para cada indicador, las cuales pueden estar desagregadas en varios grupos poblacionales.

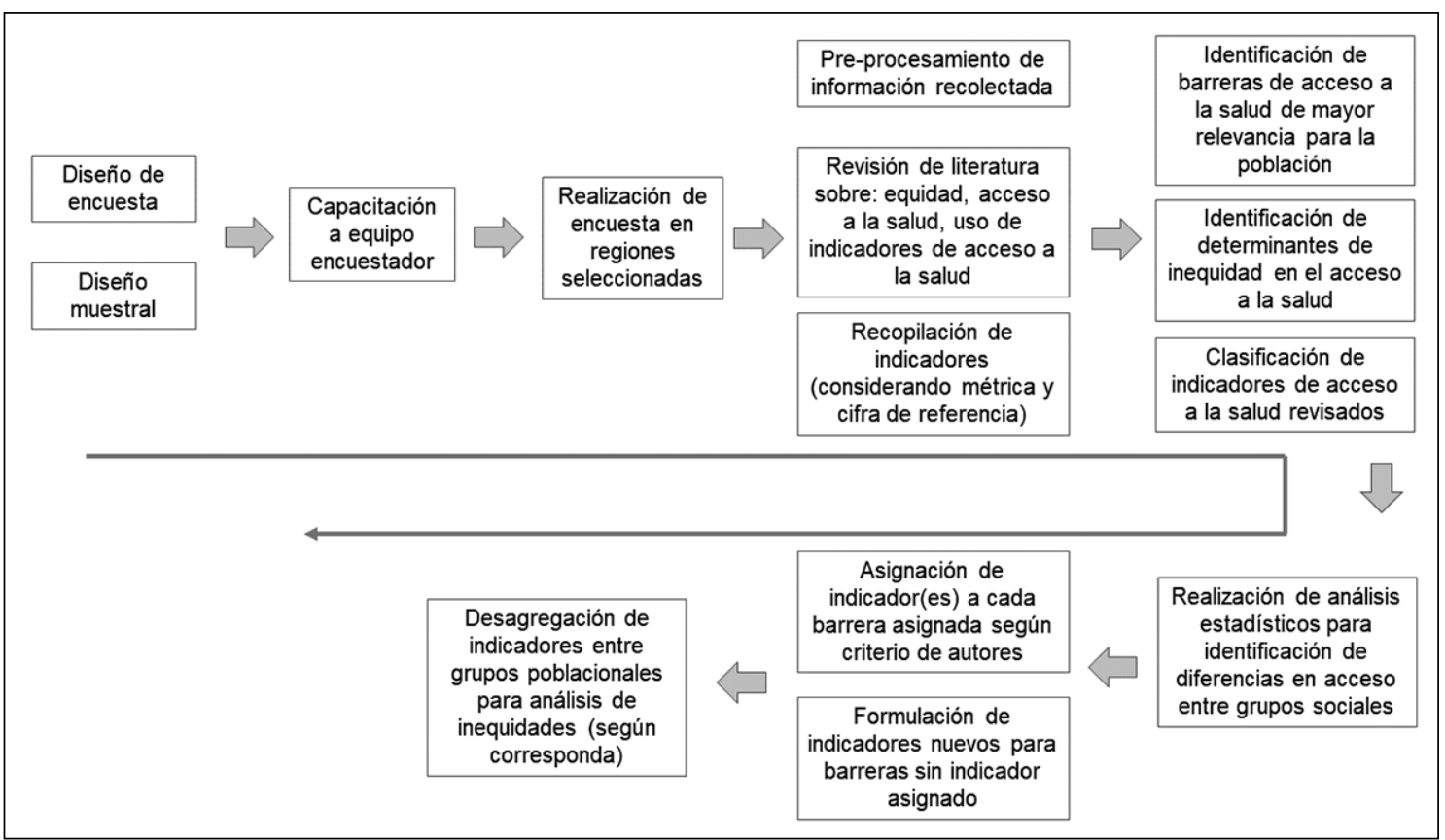

Figura 1.

Tabla 1. Barreras de acceso identificadas en la población consultada, según dimensión del modelo de acceso a la salud de Aday y Andersen

\begin{tabular}{|lccc|}
\hline Barrera & Dimensión & $\begin{array}{c}\text { Diferencias de } \\
\text { impacto entre } \\
\text { grupos }\end{array}$ & $\begin{array}{c}\text { Análisis estadísticos realizados para } \\
\text { identificar diferencias entre grupos }\end{array}$ \\
$\begin{array}{l}\text { Inexistencia de políticas de } \\
\text { mejora de acceso a la salud }\end{array}$ & Política de Salud & NA & \\
\hline $\begin{array}{l}\text { Alcance de las comunicaciones } \\
\text { de promoción en salud }\end{array}$ & Política de Salud & $\begin{array}{c}\text { Quintil de ingreso } \\
\text { Nivel educativo } \\
\text { Rango etario }\end{array}$ & $\begin{array}{l}\text { Prueba de diferencia de proporciones de } \\
\text { conocimiento de programas de salud entre } \\
\text { regiones y quintil de ingreso }\end{array}$ \\
\hline $\begin{array}{l}\text { Nivel de conocimiento de la } \\
\text { población de los programas y } \\
\text { políticas de salud }\end{array}$ & Política de Salud & $\begin{array}{l}\text { Región de } \\
\text { residencia }\end{array}$ & $\begin{array}{l}\text { Regresión logística para identificar relación } \\
\text { entre variables dependientes en probabilidad } \\
\text { de conocimiento de los programas de salud }\end{array}$ \\
$\begin{array}{l}\text { Distancia del hogar al centro de } \\
\text { salud más cercano }\end{array}$ & $\begin{array}{l}\text { Características del } \\
\text { Servicio de Salud }\end{array}$ & $\begin{array}{c}\text { Ámbito } \\
\text { rural-urbano }\end{array}$ & $\begin{array}{l}\text { Prueba de diferencia de proporciones de } \\
\text { disponibilidad de establecimientos de salud } \\
\text { entre residentes en zonas rurales y urbanas }\end{array}$ \\
\hline
\end{tabular}


Tabla 1. Barreras de acceso identificadas en la población consultada, según dimensión del modelo de acceso a la salud de Aday y Andersen (continuación)

\begin{tabular}{|c|c|c|c|}
\hline Barrera & Dimensión & $\begin{array}{l}\text { Diferencias de } \\
\text { impacto entre } \\
\text { grupos }^{1}\end{array}$ & $\begin{array}{l}\text { Análisis estadísticos realizados para } \\
\text { identificar diferencias entre grupos }\end{array}$ \\
\hline $\begin{array}{l}\text { Disponibilidad de consultorios } \\
\text { generales de salud }\end{array}$ & $\begin{array}{l}\text { Características del } \\
\text { Servicio de Salud }\end{array}$ & $\begin{array}{l}\text { Ámbito rural- } \\
\text { urbano } \\
\text { Región de } \\
\text { residencia }\end{array}$ & $\begin{array}{l}\text { Prueba de diferencia de proporciones de la } \\
\text { población de cada región que reporta que } \\
\text { el establecimiento de salud en mención } \\
\text { está disponible desde su hogar, según cada } \\
\text { par de regiones }\end{array}$ \\
\hline $\begin{array}{l}\text { Tiempos de espera entre } \\
\text { obtención de cita hasta } \\
\text { realización de consulta }\end{array}$ & $\begin{array}{l}\text { Características del } \\
\text { Servicio de Salud }\end{array}$ & $\begin{array}{l}\text { Región de } \\
\text { residencia } \\
\text { Subsistemas } \\
\text { público y privado }\end{array}$ & $\begin{array}{l}\text { Prueba de la mediana de distribución de } \\
\text { respuestas sobre tiempos de espera por } \\
\text { región y por subsistema de salud (público } \\
\text { o privado) }\end{array}$ \\
\hline $\begin{array}{l}\text { Tiempos de espera en el } \\
\text { establecimiento de salud }\end{array}$ & $\begin{array}{l}\text { Características del } \\
\text { Servicio de Salud }\end{array}$ & $\begin{array}{c}\text { Tipo de } \\
\text { establecimiento } \\
\text { público o privado }\end{array}$ & $\begin{array}{l}\text { Significancia de diferencias en distribuciones } \\
\text { de respuestas según prueba } U \text { de Mann } \\
\text { Withney entre usuarios de establecimientos } \\
\text { públicos y privados de salud }\end{array}$ \\
\hline $\begin{array}{l}\text { Tiempos de traslado al } \\
\text { establecimiento de salud }\end{array}$ & $\begin{array}{l}\text { Características del } \\
\text { Servicio de Salud }\end{array}$ & NA & \\
\hline $\begin{array}{l}\text { Características demográficas } \\
\text { relevantes }\end{array}$ & $\begin{array}{l}\text { Características de } \\
\text { la Población }\end{array}$ & NA & \\
\hline $\begin{array}{l}\text { Determinantes sociales de la } \\
\text { salud }\end{array}$ & $\begin{array}{l}\text { Características de } \\
\text { la Población }\end{array}$ & NA & \\
\hline $\begin{array}{l}\text { Cobertura de aseguramiento } \\
\text { de salud }\end{array}$ & $\begin{array}{l}\text { Características de } \\
\text { la Población }\end{array}$ & NA & \\
\hline $\begin{array}{l}\text { Presencia de condiciones } \\
\text { preexistentes y crónicas de salud }\end{array}$ & $\begin{array}{l}\text { Características de } \\
\text { la Población }\end{array}$ & NA & \\
\hline Número de consultas médicas & $\begin{array}{l}\text { Utilización de } \\
\text { Servicios de Salud }\end{array}$ & NA & \\
\hline $\begin{array}{l}\text { Niveles de necesidades no } \\
\text { satisfechas en salud }\end{array}$ & $\begin{array}{l}\text { Utilización de } \\
\text { Servicios de Salud }\end{array}$ & $\begin{array}{l}\text { Rango etario } \\
\text { Género } \\
\text { Quintil de ingresos } \\
\text { Tipo de } \\
\text { aseguramiento }\end{array}$ & $\begin{array}{l}\text { Regresión logística para identificar } \\
\text { relación entre variables dependientes en } \\
\text { probabilidad de tener necesidades de salud } \\
\text { insatisfechas }\end{array}$ \\
\hline $\begin{array}{l}\text { Acceso a medicamentos según } \\
\text { asequibilidad y disponibilidad }\end{array}$ & $\begin{array}{l}\text { Utilización de } \\
\text { Servicios de Salud }\end{array}$ & $\begin{array}{l}\text { Quintil de ingresos } \\
\text { Región de } \\
\text { residencia }\end{array}$ & $\begin{array}{l}\text { Pruebas de diferencias de proporciones de } \\
\text { niveles de presencia de barrera de acceso } \\
\text { por costos altos de medicamentos, entre } \\
\text { quintiles de ingreso y entre regiones }\end{array}$ \\
\hline $\begin{array}{l}\text { Percepción de justicia por } \\
\text { valores pagados por el sistema } \\
\text { previsional de salud }\end{array}$ & $\begin{array}{l}\text { Satisfacción del } \\
\text { Usuario }\end{array}$ & $\begin{array}{l}\text { Quintil de ingresos } \\
\text { Tipo de } \\
\text { aseguramiento }\end{array}$ & $\begin{array}{l}\text { Prueba de la mediana de distribución de } \\
\text { respuestas sobre tiempos de espera por } \\
\text { atención médica entre pares de quintiles y } \\
\text { entre afiliados a sistema privado o público } \\
\text { de salud }\end{array}$ \\
\hline Comunicación médico-paciente & $\begin{array}{l}\text { Satisfacción del } \\
\text { Usuario }\end{array}$ & NA & \\
\hline $\begin{array}{l}\text { Duración de las consultas } \\
\text { médicas }\end{array}$ & $\begin{array}{l}\text { Satisfacción del } \\
\text { Usuario }\end{array}$ & NA & \\
\hline $\begin{array}{l}\text { Satisfacción con servicio } \\
\text { brindado por el médico tratante }\end{array}$ & $\begin{array}{l}\text { Satisfacción del } \\
\text { Usuario }\end{array}$ & NA & \\
\hline $\begin{array}{l}\text { Satisfacción con el servicio } \\
\text { brindado por el establecimiento } \\
\text { de salud }\end{array}$ & $\begin{array}{l}\text { Satisfacción del } \\
\text { Usuario }\end{array}$ & NA & \\
\hline
\end{tabular}

${ }^{1}$ Se consideró como inequidad toda diferencia estadísticamente significativa entre los distintos sub-grupos sociales considerando desde un valor de $p<0,1$. 


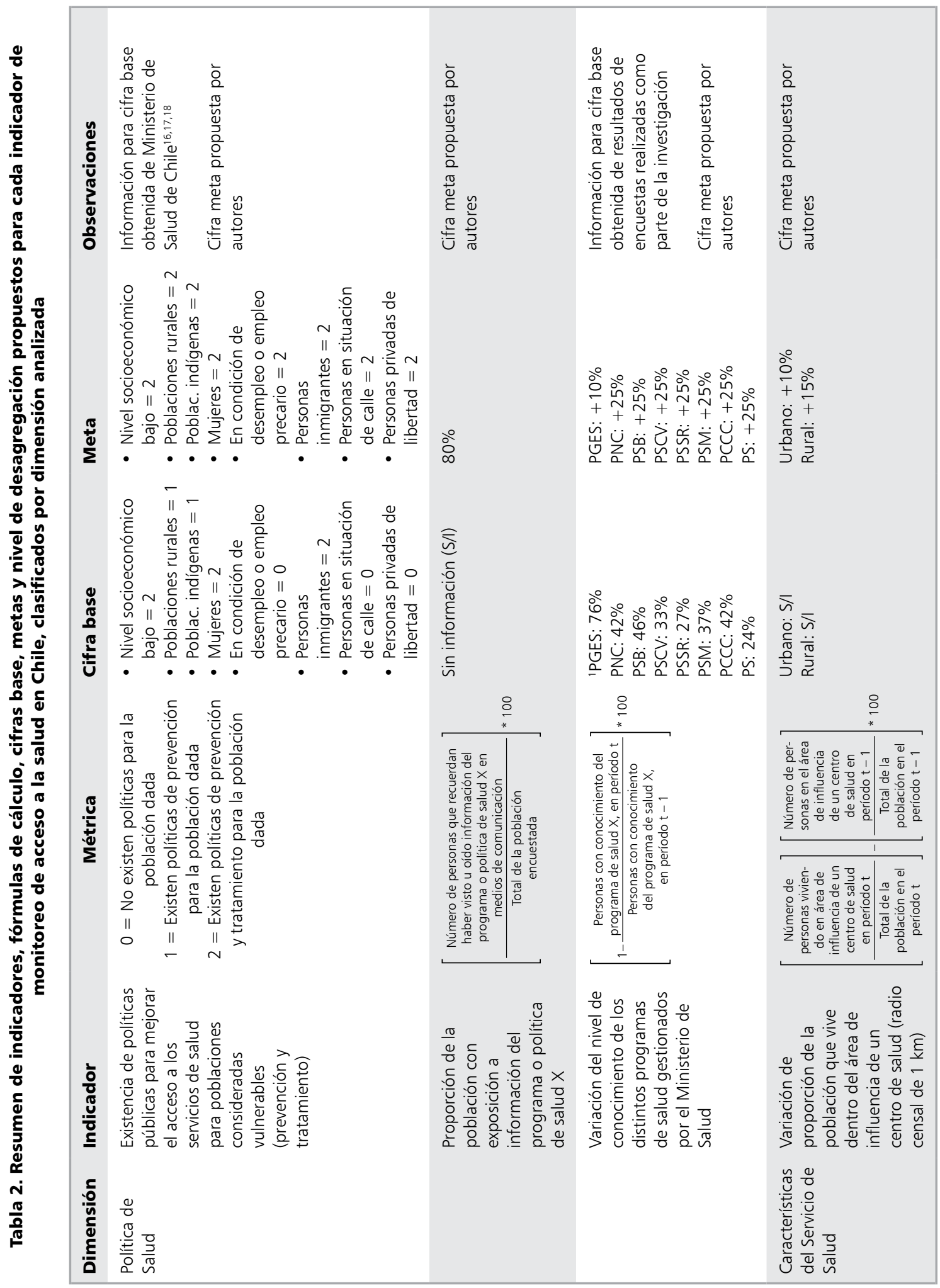




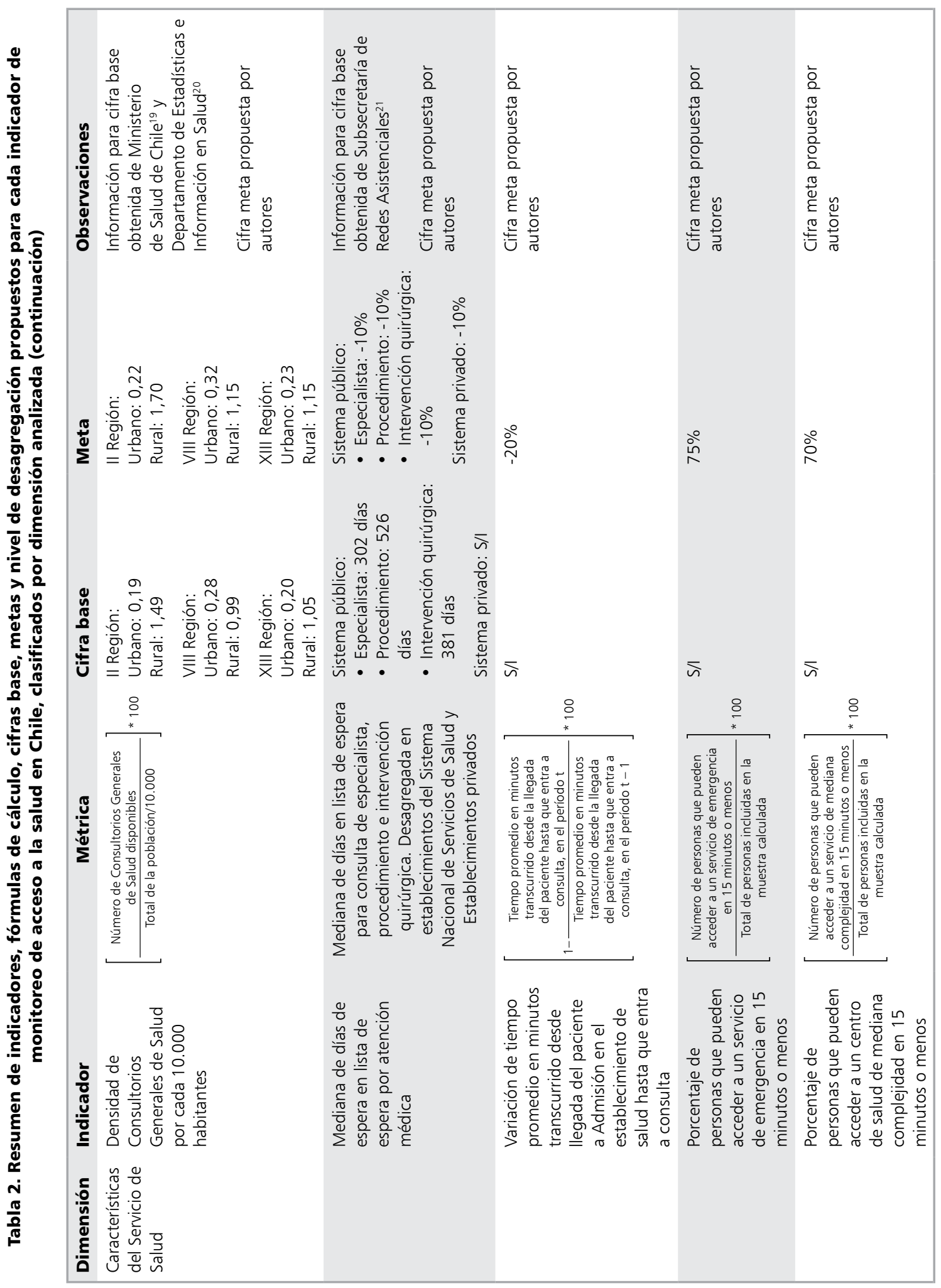




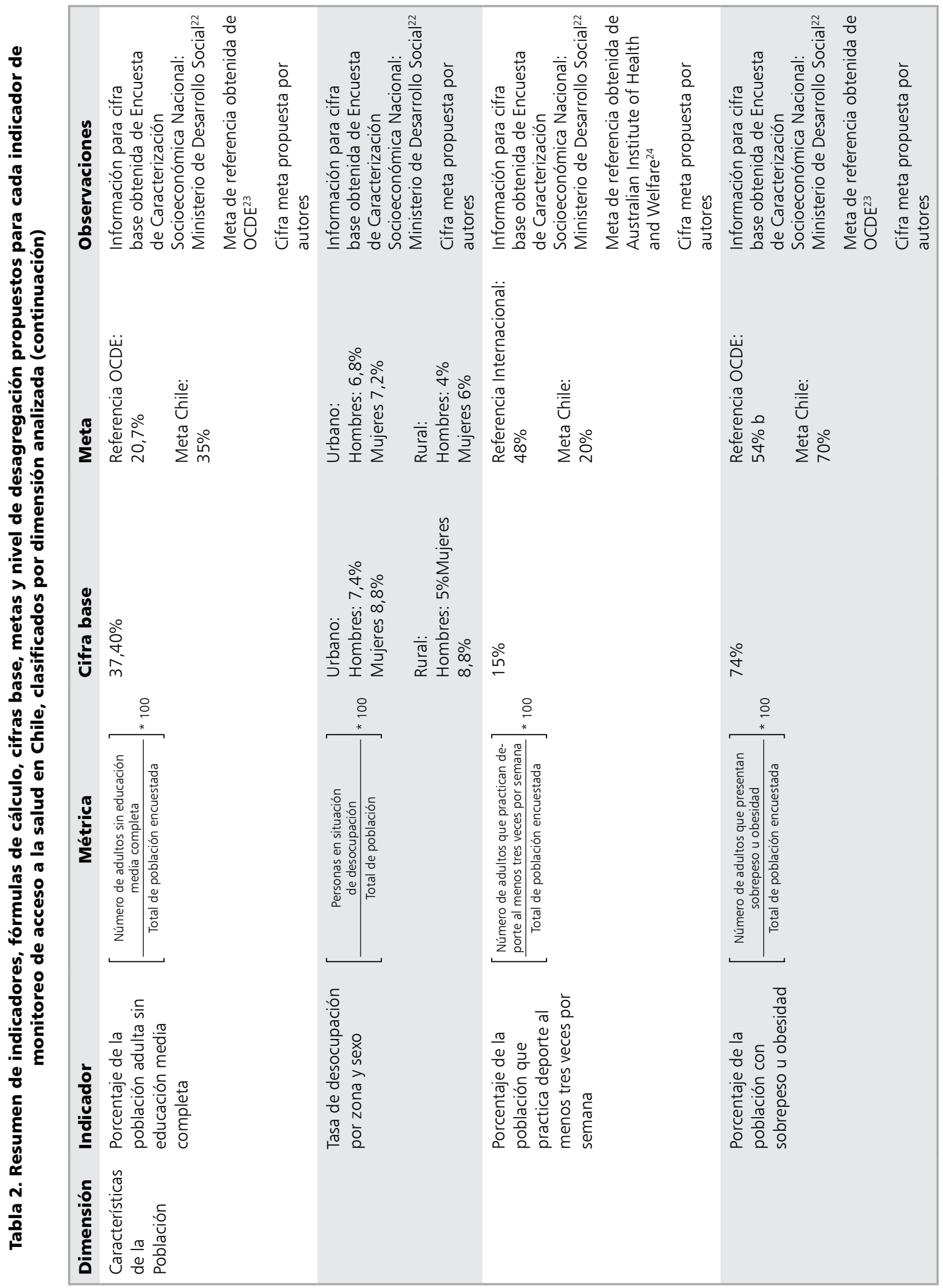




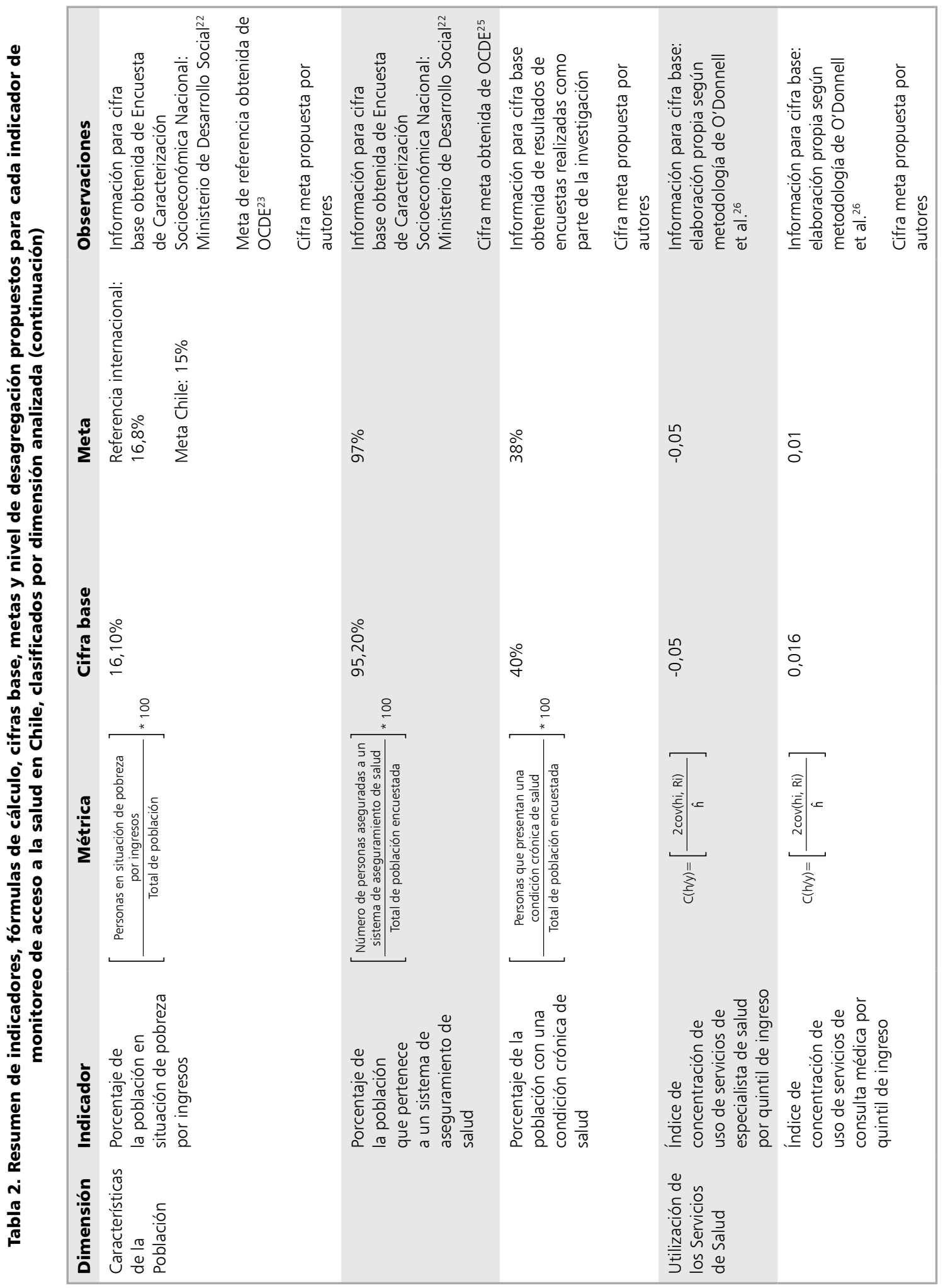




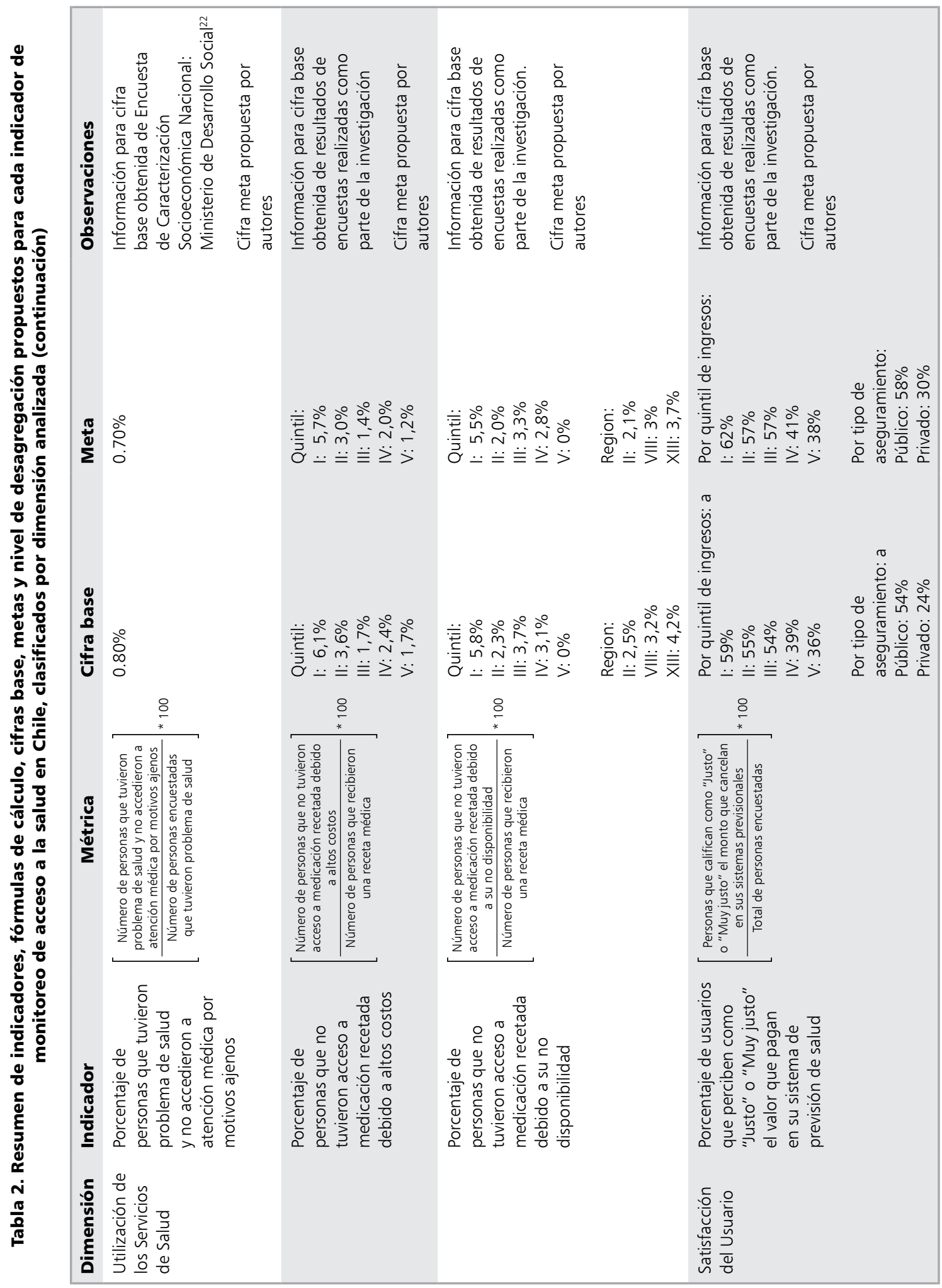




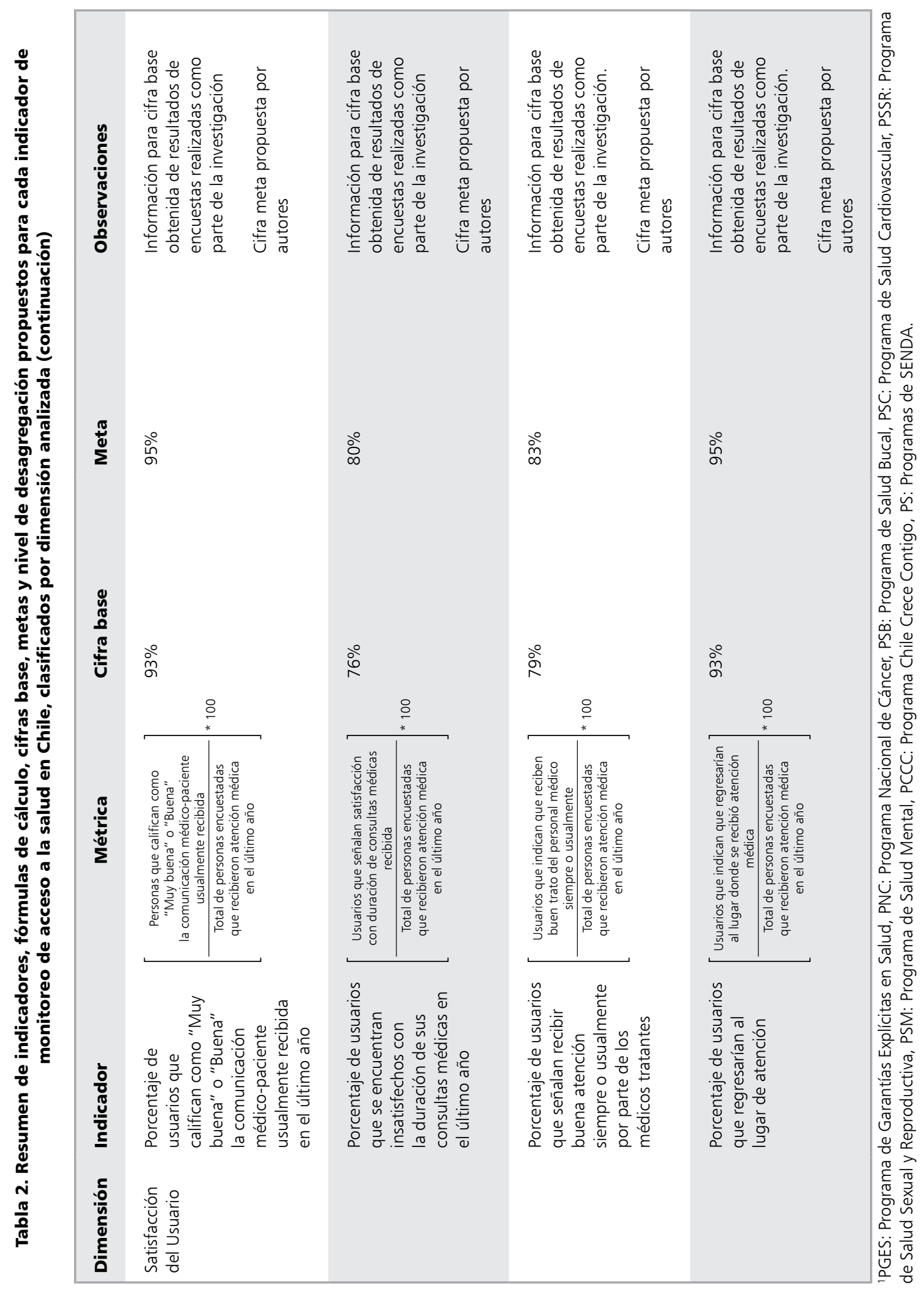




\section{Discusión}

Este estudio propone un sistema de 26 indicadores que busca monitorear las barreras de acceso a la salud que las poblaciones de las regiones analizadas reportaron. Se ha seguido un modelo lógico de entradas, procesos y resultados traducido en cinco dimensiones de acceso a la salud.

Este modelo complementa los indicadores de monitoreo actuales en Chile y propone una relación entre los resultados de las dimensiones de política de salud, características del servicio de salud y características de la población y las dimensiones de utilización de servicios de salud y satisfacción de los usuarios. Con esto se busca, bajo un enfoque de control de gestión, no solo monitorear indicadores de utilización o satisfacción con servicios de salud, sino vigilar aquellos elementos determinantes que influencian dichos indicadores de resultados a fin de poder tomar acción como parte de la estrategia sanitaria.

El estudio incluyó la realización de análisis estadísticos que permitan identificar inequidades entre distintos grupos sociales para cada uno de los elementos considerados como barreras de acceso que se estudiaron. Al analizar los factores determinantes de una potencial inequidad en el acceso entre grupos sociales en la muestra recolectada, se observan patrones previamente citados en la literatura; por ejemplo, el impacto de residir en una comunidad rural con una menor accesibilidad geográfica a los servicios de salud, la influencia del nivel de ingresos en la probabilidad de recibir atención médica y de tener una mayor satisfacción con los servicios de salud, o cómo tener un seguro privado de salud permite esperar menos tiempo para recibir atención médica. Dichos hallazgos sirvieron para proponer que los resultados de cada indicador sean desagregados en los distintos grupos sociales que sufren inequidad en cada elemento, a fin de realizar un monitoreo más específico en busca de una mejora en el acceso bajo un enfoque de equidad. El presente estudio contribuye a la literatura existente al proponer indicadores basados en un modelo ontológico realizado específicamente para el mapeo de barreras de acceso a la salud, mientras que la mayoría de estudios existentes, tal como mencionan Jimenez, Barriga y Salazar ${ }^{27}$ parten de la información obtenida en el módulo de salud de la encuesta CASEN para el análisis de inequidades en el acceso a la salud.
Entre las limitaciones principales de este estudio se encuentra el hecho de que abarque únicamente tres regiones de Chile, debido a lo cual sus resultados no son generalizables al resto de regiones, cuyas poblaciones pueden indicar mayor prevalencia de otras barreras de acceso no identificadas en esta ocasión. Futuras investigaciones podrían ampliar el alcance de este análisis al resto de regiones del país y comprobar si los resultados son similares. Asimismo, este estudio se centra en la propuesta de un sistema de indicadores, quedando fuera de su alcance la aplicación práctica de este. Finalmente, a falta de información interna específica respecto a estadísticas, recursos o prioridades del sistema de salud chileno con relación al acceso, para la definición de las cifras de las metas, se han considerado metas de referencia de otros sistemas de salud nacionales, entidades supranacionales, la literatura e información obtenida de las encuestas recopiladas como parte de este estudio. Una eventual redefinición de cifras base y metas que tengan sustento en información interna referida a estadísticas históricas, recursos disponibles y capacidad de implementación permitirá obtener un sistema de indicadores con mayor solidez.

De esta forma, el impacto que tiene el presente estudio en el diseño de políticas públicas de mejora de acceso a la salud en Chile consta de dos partes: en primer lugar, identifica qué elementos, además de la necesidad de atención médica, están asociados a una mayor o menor utilización de servicios de salud, así como una mayor o menor percepción en la calidad de estos, permitiendo sentar las bases para poder tomar acción al respecto. En segundo lugar, la búsqueda, recopilación y análisis de indicadores de relevancia para cada dimensión ha permitido, para varios de los indicadores, establecer metas de referencia internacionales para identificar cuál es la posición actual de Chile. Esto es particularmente importante pues, permite indagar cuáles son las buenas prácticas realizadas en otros sistemas de salud y estudiar la factibilidad de adaptarlas a la realidad local a fin de, en un mediano o largo plazo, poder cerrar la brecha entre el valor actual y el de referencia, logrando elevar el nivel de vida de la población chilena.

\section{Declaraciones}

Financiamiento: Este estudio es parte del Pro- 
yecto del Fondo Nacional de Desarrollo Científico y Tecnológico (FONDECYT) No 11160150, recibió financiamiento de la Comisión Nacional de Investigación Científica y Tecnológica.

\section{Referencias}

1. Fineberg HV. A Successful and Sustainable Health System-How to Get There from Here. N Engl J Med 2012; 366 (11): 1020-7.

2. Observatorio Chileno de Salud Pública. Panorama y tendencias de la salud en Chile. Revisión 2013. Santiago de Chile; 2014.

3. Missoni E, Solimano G. Towards Universal Health Coverage: the Chilean experience. World Health Organization; 2010. (Health-Systems Financing).

4. Goic A. El Sistema de Salud de Chile: una tarea pendiente. Rev Med Chile 2015; 143 (6): 774-86.

5. Becerril-Montekio V, Manuel A. Sistema de salud de Chile. Salud Pública México 2011; 53: 12.

6. Jacobs B, Ir P, Bigdeli M, Annear PL, Van Damme W. Addressing access barriers to health services: an analytical framework for selecting appropriate interventions in low-income Asian countries. Health Policy Plan 2012; 27 (4): 288-300.

7. DeGroff A, Schooley M, Chapel T, Poister TH. Challenges and strategies in applying performance measurement to federal public health programs. Eval Program Plann 2010; 33 (4): 365-72.

8. Organización Panamericana de la Salud OM de la S. Estrategia para el acceso universal a la salud y la cobertura universal de salud [Internet]. Washington: Organización Mundial de la Salud; 2014 oct. Report No.: CD53/5, Rev. 2. Disponible en: https://www.paho.org/hq/dmdocuments/2014/CD53-5-s.pdf.

9. Pinzón CE, Chapman E, Panisset U, Arredondo A, Fitzgerald J, Reveiz L. Disponibilidad de indicadores para el seguimiento del alcance de la "Salud Universal" en América Latina y el Caribe. Rev Panam Salud Pública 2016; 39: 330-40.

10. Elinson J. Toward Sociomedical Health Indicators. Soc Indic Res 1974; 1 (1): 59-71.

11. Aday LA, Andersen R. A Framework for the Study of Access to Medical Care. Health Serv Res 1974; 9 (3): 208-20.

12. Babitsch B, Gohl D, von Lengerke T. Re-revisiting Andersen's Behavioral Model of Health Services Use: a systematic review of studies from 1998-2011. GMS Psycho-Soc-Med [Internet]; 9. Disponible en: https://www. ncbi.nlm.nih.gov/pmc/articles/PMC3488807/ [citado el
10 de diciembre de 2018].

13. Núñez A, Ramaprasad A, Syn T, Lopez H. An ontological analysis of the barriers to and facilitators of access to healthcare. J Public Health [Internet]; Disponible en: http://link.springer.com/10.1007/s10389-020-01265-4 [citado el 12 de julio de 2020].

14. Andersen RM, McCutcheon A, Aday LA, Chiu GY, Bell R. Exploring dimensions of access to medical care. Health Serv Res 1983; 18 (1): 49-74.

15. World Health Organization. Setting Targets for Health 2020 [Internet]. Baku, Azerbaijan; 2011 sep. Report No.: EUR/RC61/Inf.Doc./7. Disponible en: http://www.euro. who.int/_data/assets/pdf_file/0008/149282/RC61_InfDoc7.pdf.

16. Ministerio de Salud de Chile. Estrategia nacional de salud para el cumplimiento de los objetivos sanitarios de la década 2011-2020. Santiago, Chile: MINSAL; 2011. Disponible en: http://www.bibliotecaminsal.cl/ wp/wp-content/uploads/2011/12/Metas-2011-2020. pdf.

17. Ministerio de Salud. Plan Nacional de Salud Mental 2017-2025. Ministerio de Salud; 2018 oct. Disponible en: https://www.minsal.cl/wp-content/uploads/2017/12/ PDF-PLAN-NACIONAL-SALUD-MENTAL-2017-A2025.-7-dic-2017.pdf.

18. Ministerio de Salud. Política de Salud de Migrantes Internacionales. Santiago de Chile: Ministerio de Salud; 2017. Disponible en: https://www.minsal.cl/wp-content/uploads/2015/09/2018.01.22.POLITICA-DE-SALUD-DE-MIGRANTES.pdf.

19. Instituto Nacional de Estadísticas. Compendio Estadístico 2017. 2017. Disponible en: https://www.ine.cl/ estadisticas/.

20. Departamento de Estadísticas e Información de Salud. Listado De Establecimientos [Internet]. 2018 [citado 20 de agosto de 2018]. Disponible en: https://reportesdeis. minsal.cl/ListaEstablecimientoWebSite/.

21. Subsecretaría de Redes Asistenciales. Plan Nacional de Tiempos de Espera No GES en Chile en Modelo de Atención en RISS (Redes Integradas de Servicios de Salud) 2014-2018 [Internet]. Ministerio de Salud; 2018. Disponible en: http://www.minsal.cl/wp-content/ uploads/2018/03/Plan-nacional-de-tiempos-de-esperaNo-GES.pdf.

22. Ministerio de Desarrollo Social. Encuesta de Caracterización Socioeconómica Nacional - 2017 [Internet]. 2018 [citado 11 de diciembre de 2018]. Disponible en: http:// observatorio.ministeriodesarrollosocial.gob.cl.

23. OCDE. Education at a Glance 2018: OECD Indicators. OECD Publishing; Éditions OCDE; 2018.

24. Australian Institute of Health and Welfare. National 
Health Performance Framework [Internet]. 2018 [citado 21 de agosto de 2018]. Disponible en: http://meteor. aihw.gov.au/content/index.phtml/itemId/392569.

25. OCDE. Health at a glance 2017: OECD indicators. Paris: OECD; 2017.

26. O’Donnell O, O’Neill S, Van Ourti T, Walsh B. conin- dex: Estimation of concentration indices. Stata J 2016; 16 (1): 112-38.

27. Jiménez S, Barriga O, Salazar A. Inequidad en el acceso a salud en Chile: estudio multifactorial basado en la Encuesta CASEN del año 2013. Rev Chil Salud Pública; 22 (1): 31-40. 